\title{
Entrenador virtual para el aprendizaje de rutinas motoras, en personas con ausencia de un miembro superior
}

\author{
William David Vargas $\mathrm{P}^{1}$ \\ Pablo Alexander Munévar $\mathrm{G}^{2}$ \\ John Jairo Páez $\mathrm{R}^{3}$
}

\section{Resumen}

El proyecto de investigación en el campo de las ayudas aumentativas para discapacitados, tiene como objetivo principal evaluar el aprendizaje de rutinas motoras en una persona con amputación traumática a nivel del tercio distal del codo por medio de un entrenador virtual controlado por señales mioeléctricas. Este tema de investigación es de gran importancia porque prepara a la persona para el uso de una prótesis real y porque a nivel cognitivo, el entrenador puede crear un sistema de auto-representación que se convierte en un punto de convergencia en el sistema de retroalimentación visual y propioceptiva. Además, debido a la relación de manipulación identificación - transformación de objetos, ofrece al cerebro nuevos modos de aproximarse a tareas anteriores y la posibilidad de emprender otras nuevas. Para esta investigación se diseñó, elaboró y validó el sistema de captura y reconocimiento de las señales mioeléctricas y el modelo de aprendizaje por observación propuesto inicialmente.

\footnotetext{
1 Licenciado en Diseño Tecnológico, Universidad Pedagógica Nacional (2010). Tecnólogo en Ingeniería Mecatrónica de la Universidad Distrital Francisco José de Caldas (2005). Estudios actuales de Ingeniería en Control en la Universidad Distrital Francisco José de Caldas. Email: wupntco@misena.edu.co.

2 Licenciado en Electrónica, Universidad Pedagógica Nacional (2000). Magíster en Educación, Universidad Pedagógica Nacional (2008). Doctorando en Educación, Currículo Profesorado e Instituciones Educativas, Universidad de Granada (España). Docente de tiempo completo. Escuela Ciencias de la Educación. Universidad Nacional Abierta y a Distancia. Email: pablo.munevar@unad.edu.co.

3 Licenciado en Diseño Tecnológico, Universidad Pedagógica Nacional (2003). Magister en Tecnologías de la Información Aplicadas a la Educación, Universidad Pedagógica Nacional (2008). Candidato a Magister en Ingeniería de Sistemas en la Pontificia Universidad Javeriana de Bogotá. Docente de tiempo completo, Facultad de Ciencias y Educación, Universidad Distrital Francisco José de Caldas. Email: johnpa@live. com.
} 
Palabras clave: Aprendizaje de rutinas motoras, discapacidad, realidad virtual, señales mioeléctricas, ambientes virtuales.

\title{
A virtual coach to train people missing an upper limb in motor routines
}

\begin{abstract}
This research project in the field of augmentative aids for disabled people has as its main objective the assessment of motor routines training in persons with traumatic amputation on the distal side by a virtual trainer controlled by myoelectric signals. This research study is important, since it prepares the individual for the use of a real prosthesis and, thus, on a cognitive level, the virtual coach can create a system of self-representation, which is a point of convergence in the visual feedback system and is proprioceptive. In addition, because of the relationship of manipulationidentification-transformation of objects, the brain provides new ways of approaching prior tasks and the possibility of starting new ones. For this investigation, a system for capturing and recognizing myoelectric signals and the observational learning model proposed was designed, developed and validated.
\end{abstract}

Keywords: Motor learning routines, disability, virtual reality, myoelectric signals, virtual environments.

Recibido: Octubre de 2010

Aceptado: Enero 11 de 2011

\section{Introducción}

El proyecto de investigación en el campo de las ayudas aumentativas para discapacitados, se enfoca en el aprendizaje de rutinas motoras en procesos prensiles, por medio de un entorno virtual. El aprendizaje de estas rutinas es de gran importancia a nivel cognitivo, dado que la mano permite crear un sistema de autorepresentación, es un punto de convergencia en el sistema de retroalimentación visual y propioceptiva, debido a la relación de manipulación —identificacióntransformación de objetos y a que ofrece al cerebro nuevos modos de aproximarse a tareas anteriores y la posibilidad de emprender otras nuevas. 
Wilson (1998) afirma que en los seres humanos la inteligencia ha estado relacionada con el diseño y construcción de artefactos. La mano ha sido la compañera fundamental del cerebro para convertir el pensamiento en acción, ya que las ideas se traducen mecánicamente en acciones, estimulando así en el cerebro la construcción de nuevos programas motores, rediseñando caminos sinápticos, procesando datos visuales para crear formas de movimiento, cambiando planos de referencia y creando representaciones jerárquicas para configurar los procesos perceptuales útiles en el control de movimientos. En otras palabras, el cerebro le habla a la mano como la mano le habla al cerebro.

Uno de los problemas en el proceso de adaptación de las prótesis mioeléctricas es el esfuerzo cognitivo que tiene que hacer el sujeto durante su apropiación y aprendizaje. Por este motivo surge la siguiente pregunta de investigación: ¿Cuáles son las características técnicas y pedagógicas necesarias para el desarrollo de una herramienta de representación virtual de la mano humana controlada por señales mioeléctricas, en personas con amputación traumática de miembro superior derecho?

\section{Metodología}

Módulo de realidad virtual de Matlab aplicado al simulador de prótesis virtual mioeléctrico

La manera de elaborar la cinemática directa por el método cinemático de Denavit - Hartenberg para la mano humana, se fundamenta en las ecuaciones de movimiento y se representa el movimiento general del entrenador mediante Matlab. Se debe implementar una interfaz gráfica en donde se busca representar visualmente la mano mediante un programa de diseño tridimensional para lograr visualizar bien los movimientos a ejecutar y conseguir una apariencia un poco más realista de la mano.

En la actualidad se tienen diferentes medios de programación en los cuales se puede simular mundos virtuales. Es el caso de programas como VrmlPad, 3D ImageScene, Cosmo Player entre otros, en los que se busca simular entornos en tiempo real, para la representación de la mano humana. En este caso se labora en un programa de realidad virtual el cual tiene integrado Matlab, cuyo funcionamiento es compatible con la programación de las ecuaciones de movimiento. V-Realm-Builder es una herramienta gráfica para la edición 3D que proporciona una interfaz con sintaxis de VRML. ${ }^{4}$ Una de las características más importantes de este programa es que al estar integrado con Matlab, se puede trabajar paralelamente para la simulación, en función de una programación 


\section{William David Vargas, Pablo Alexander Munévar y John Jairo}

Entrenador virtual para el aprendizaje de rutinas motoras, en personas con ausencia de un miembro superior, artículo producto de la investigación

establecida. A continuación, en las figuras 2, 3, 4, 5, 6 y 7 se presentan los cinco movimientos que se han desarrollado en Matlab.

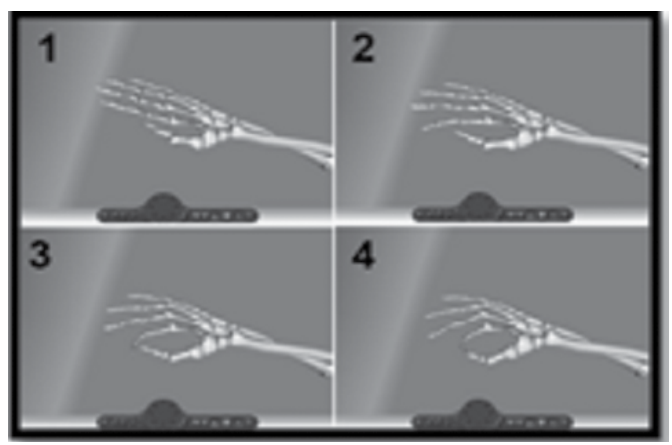

Figura 1. Simulación del movimiento de pinza bidigital de la mano en función de las ecuaciones de movimiento elaboradas en Matlab. Fuente: El presente estudio.

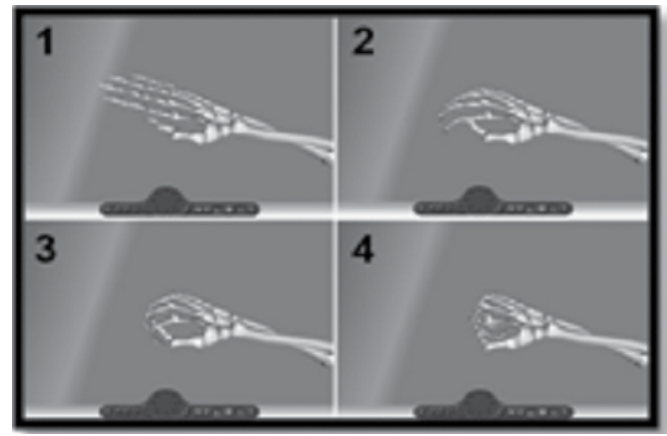

Figura 2. Simulación del movimiento de cierre de la mano en función de las ecuaciones de movimiento elaboradas en Matlab. Fuente: El presente estudio

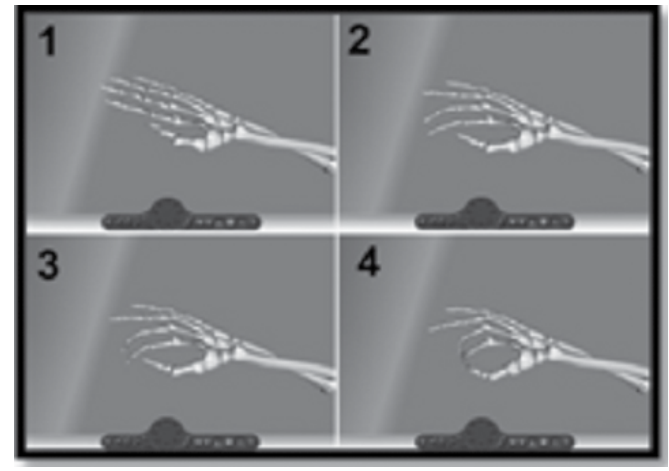

Figura 3. Simulación del movimiento de pinza tridigital de la mano en función de las ecuaciones de movimiento elaboradas en Matlab. Fuente: El presente estudio. 


\section{Revista de}

investigaciones UNAD

Volumen 10. Número 2. Diciembre 2011

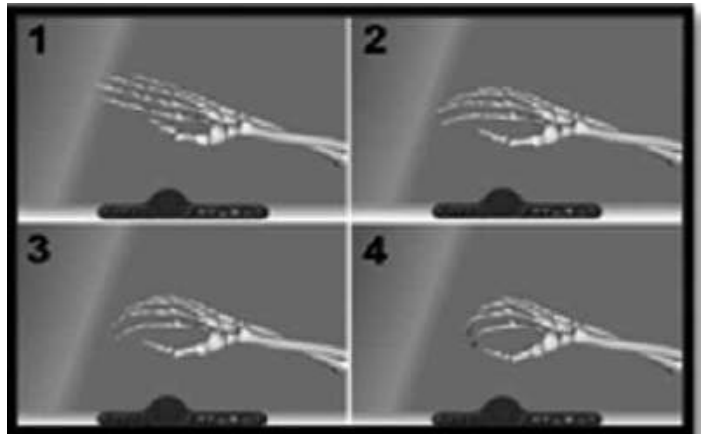

Figura 4. Simulación del movimiento de agarre cilíndrico en función de las ecuaciones de movimiento elaboradas en Matlab. Fuente: El presente estudio.

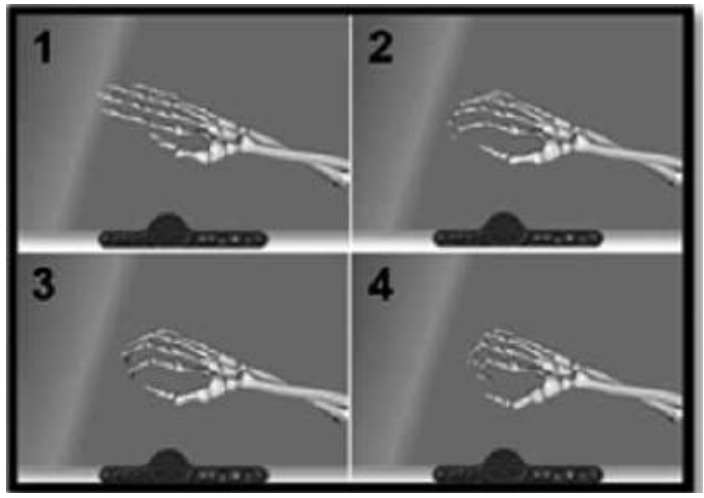

Figura 5. Simulación del movimiento de agarre tipo gancho de la mano en función de las ecuaciones de movimiento elaboradas en Matlab. Fuente: El presente estudio.

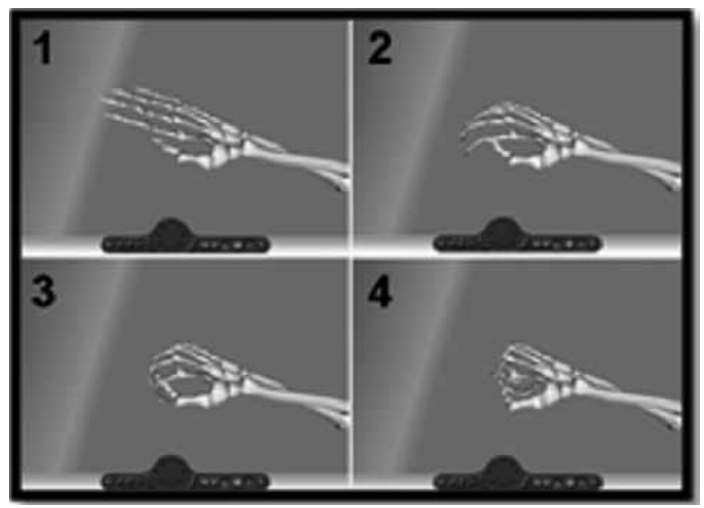

Figura 6. Simulación del movimiento de cierre de la mano en función de las ecuaciones de movimiento elaboradas en Matlab. Fuente: El presente estudio. 
El desarrollo de este proyecto se enmarca en el modelo de investigación cualitativa - experimental, donde se pretende evaluar un ambiente de aprendizaje de rutinas motoras en un sujeto con amputación de miembro superior derecho a nivel del tercio proximal del antebrazo, cuando este controla una simulación del miembro superior en un programa de realidad virtual soportado en el software Matlab, mediante señales generadas por el cuerpo humano.

Para la evaluación del sistema de aprendizaje se ha desarrollado una interfaz gráfica en donde se visualiza la mano humana con 15 grados de libertad y con cinco tipos de prensión básicos. Desde el punto de vista físico se representa visualmente al sujeto mediante la pantalla del computador y desde el punto de vista pedagógico se utiliza el ambiente de aprendizaje por observación, el cual concibe el desarrollo psicomotor como una organización jerarquizada que va desde los desplazamientos exógenos, pasando por los desplazamientos autónomos y llega hasta los desplazamientos corporales constructivos. (Wallon, 1997).

La metodología escogida para el desarrollo de esta propuesta es la de análisis de protocolos verbales. Según Maldonado (2001), en esta se intenta un acercamiento al estudio de la representación de conocimiento de validez general que puede ser aplicado a los procesos cognitivos de personas que tienen limitaciones sensoriales. Por esta razón es una metodología propicia para el análisis del ambiente de aprendizaje por observación en una persona con una amputación.

En esta metodología se consideran tres etapas principales. La primera es la recolección de la información que se verá representada en la experimentación y en el desarrollo de los protocolos verbales recitados por la persona que elabora el movimiento planteado. Hay que recordar que en esta experimentación la persona hace la verbalización a medida que se hace el proceso de pensamiento, lo cual se conoce como modelo de informe verbal concurrente, ejercicio que exige la reestructuración y la configuración de relaciones ante nuevas situaciones de presión (Páez, 2008). La segunda etapa es la codificación de la información, que se elabora estudiando y analizando un registro de la verbalización que previamente ha sido registrada en un formato de video para evidenciar el proceso de aprendizaje en función de los pasos utilizados por él para el desarrollo de la situación problema. Por último, en la tercera etapa se debe presentar de manera formal la información en forma de proposiciones. Este paso constituye un modelo computacional en el cual se pueden generar nuevas preguntas que amplían la comprensión del fenómeno (Maldonado, 2001).

Para el desarrollo de la propuesta se cuenta con una persona mayor de edad con una amputación ocurrida hace más de 17 años, a nivel del tercio proximal de antebrazo, de género masculino, con una diagnosis en términos de movilidad 
parcial del codo, funciones mentales globales y específicas normales, y funciones sensoriales de voz y habla normales.

El procedimiento comienza por entrenar al sujeto en el ambiente de aprendizaje por observación con las actividades correspondientes a dicho ambiente; luego se plantean cinco ejercicios de prensión para que la persona los ejecute y se pueda así definir la estrategia cognitiva utilizada para el desarrollo de la tarea.

El modelo teórico para la implementación de los protocolos verbales (Maldonado, 2001), está fundamentado en la teoría del procesamiento de la información cuyos parámetros generales son:

1. Existe un conjunto de elementos llamados símbolos.

2. La estructura de los símbolos depende de la conexión de las relaciones.

3.La memoria se considera como un elemento que almacena y coordina estructuras.

4. El proceso de información inherente al desarrollo de la actividad tiene en cuenta las estructuras de los símbolos antes y después del proceso.

5. El procesamiento de información requiere un elemento denominado procesador, el cual toma los procesos elementales de información, los combina y los prepara para interpretarlos.

El proceso de información se desarrolla en un ambiente de aprendizaje que está conformado por un conjunto de rasgos o estímulos externos que se reflejan en la representación que la persona se hace de la tarea. En este proceso hay que considerar:

1. La distribución de la información. (Punto de partida, Elaboración y Punto de llegada).

2. La naturaleza de las restricciones. (Alternativas de prensión de la prótesis y objetos geométricos presentados durante la experimentación).

3. El espacio del problema. (Representación que la persona hace de la tarea).

4. Complejidad de los problemas. (Cantidad de rutinas motoras utilizadas en su solución).

5. Proceso de transferencia. (Utilización de las rutinas aprendidas en la solución de nuevos problemas). 
6. Tipo de respuestas. (Alternativas de utilización de la prótesis por parte de la persona durante la solución de un problema de prensión).

7. Retroalimentación. (Procesos meta-cognitivos en la búsqueda de alternativas en la solución de un problema de prensión).

8. Costo de errores. (En el éxito o fracaso en la solución de un problema de prensión).

El paso siguiente en la metodología de análisis de protocolos verbales consiste en transcribir el contenido del registro de la manera más fiel posible, lo que se denomina proceso de codificación. A continuación se indican las etapas principales:

1. Transcripción de los datos grabados en formato digital.

2. Análisis de vocabulario. A continuación se relacionan los elementos conceptuales que, con base en la teoría de protocolos verbales, permiten identificar la estrategia utilizada por el sujeto durante el aprendizaje de las actividades motrices. Se presenta, además, una descripción de cada uno de ellos.

2.1 Objetos. Está relacionado con los elementos que el sujeto puede manipular con la prótesis. Por ejemplo, cilindros, esferas, cubos, etc.

2.2 Relaciones: Este descriptor permite identificar los procesos que hace el sujeto para seleccionar diferentes rutinas motrices de acuerdo con los objetos que esté manipulando y con la tarea de prensión que eventualmente desee implementar.

2.3 Operadores: Se pueden entender como la acción que al aplicarla a los objetos, cambia su estado. Por ejemplo, puede generar cambios en la posición y en la velocidad de los objetos manipulados.

2.4 Control: Está relacionado con el conocimiento que tiene el sujeto acerca de los alcances cinemáticos y dinámicos de la prótesis durante el desarrollo de las diferentes actividades motrices.

2.5 Procesos evaluativos: Procedimientos de metacognición sobre lo que hace con la prótesis.

2.6 Objetivos: marca la dirección de la acción de prensión.

3. Segmentación y codificación. Están relacionados con el acto de tomar los elementos del análisis de vocabulario para identificar la estrategia 
de trabajo que adopta el sujeto durante el aprendizaje de las actividades motrices. "Es algo así como identificar los procesos lógicos que sigue el sujeto para desarrollar las diferentes tareas".

\subsection{Codificación de Segmentos (Unidades Significativas).}

3.2 Duración.

3.3 Codificación en términos de lógica formal.

3.4 Sistema de producción (Identificación de la estrategia).

\section{Análisis de datos}

Teniendo en cuenta el enfoque del ambiente de aprendizaje utilizado, se diseña un conjunto de actividades de prensión para el desarrollo de la investigación. Como en este caso se utiliza el ambiente de aprendizaje por observación, inicialmente se hace una explicación visual y oral de los movimientos propuestos por el investigador, para que la persona los realice con la simulación del miembro superior en la pantalla del ordenador. Después se dan las instrucciones para que la persona logre hacer cinco movimientos en orden aleatorio utilizando las rutinas de prensión aprendidas. En la fase de evaluación se consideran los siguientes parámetros:

Éxito del ejercicio: Es el cumplimiento de una tarea de prensión propuesta.

Fracaso del ejercicio: No cumplimiento de una tarea de prensión propuesta.

Eficacia del ejercicio: Es la relaci ón entre el número de éxitos a alcanzar de la tarea y el número total de repeticiones.

La condición para que la persona vaya avanzando en el desarrollo de los ejercicios es que haga un mismo movimiento cinco veces. De lo contrario, deberá volver a intentarlo hasta que cumpla con el objetivo.

\section{Resultados}

En la primera etapa del ambiente de aprendizaje por observación se busca informar al sujeto el funcionamiento general del entrenador, las restricciones que existen y los tiempos que el sistema reconoce para obtener una contracción válida. Además se procede a distribuir los electrodos en los músculos de mayor actividad mioeléctrica, como se muestra a continuación: 
Entrenador virtual para el aprendizaje de rutinas motoras, en personas con ausencia de un miembro superior, artículo producto de la investigación

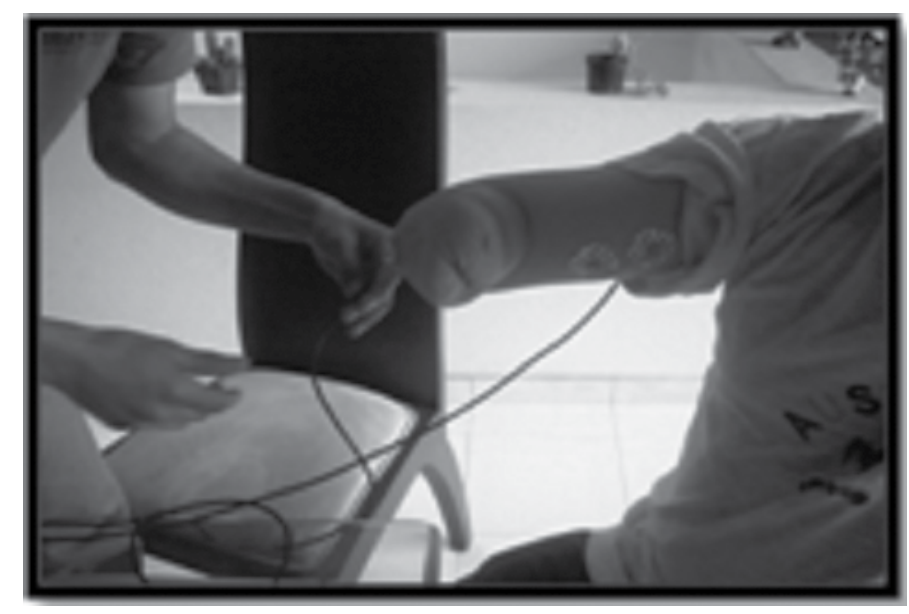

Fotografía 1. Posición de los electrodos. Fuente: El presente estudio

Según el esquema que se ha planteado para la elaboración de la experimentación, en la segunda etapa se hace una explicación visual y oral de los movimientos exógenos planteados. Por medio de la observación y una explicación verbal por parte del experimentador, la persona comprende cómo se controla la imagen. De esta manera se obtuvo la siguiente relación:

Pinza bidigital: una contracción muscular.

Pinza tridigital: dos contracciones musculares.

Agarre cilíndrico: tres contracciones musculares.

Agarre en forma de gancho: cuatro contracciones musculares.

Cierre de mano: cinco contracciones musculares.

En esta primera parte de la experimentación el objetivo principal es que la persona aprenda a hacer los movimientos de la lista anterior. En este entrenamiento la persona aprende a hacer un cierre en pinza bidigital en el simulador. Para poder establecer si la persona aprendió el movimiento, debe hacer consecutivamente cinco repeticiones correctas de cada movimiento.

De lo contrario, debe comenzar el ejercicio nuevamente hasta que lo pueda lograr.

La fotografía 2 presenta el entrenamiento de la persona durante el aprendizaje de la rutina motora para el movimiento de pinza bidigital. 


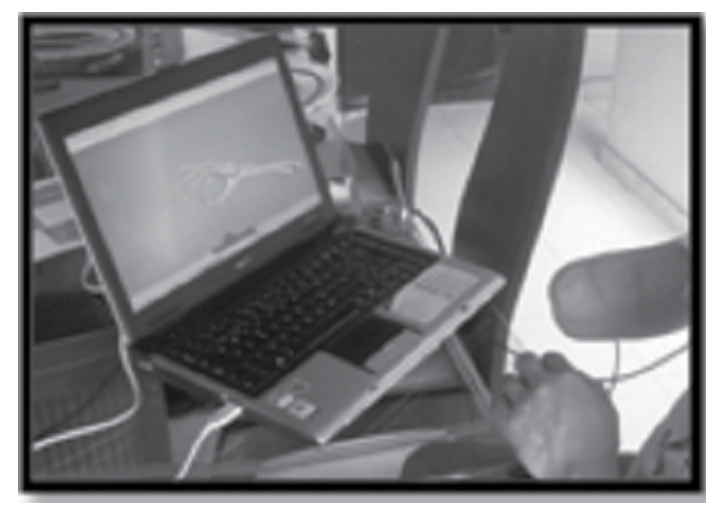

Fotografía 2. Entrenamiento del movimiento de pinza bidigital. Fuente: El presente estudio.

A medida que se va avanzando en los ejercicios, el nivel de complejidad va aumentando, dado que la cantidad de contracciones es mayor para la generación de cada movimiento. Es interesante analizar cómo a medida que aumenta la curva de aprendizaje, la persona va automatizando los movimientos y generándolos de manera más rápida.

En la Figura 1 se presenta el resumen de los intentos realizados en cada movimiento $\mathrm{v} / \mathrm{s}$ los cinco movimientos:

\section{Progreso en los ejercicios Fase 1}

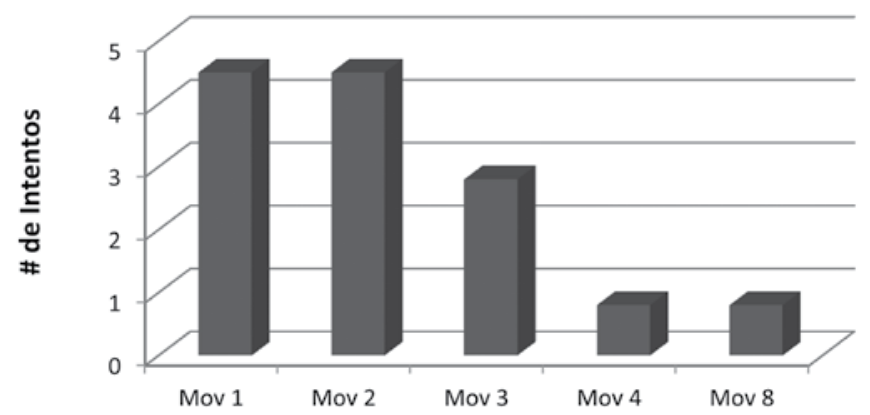

Figura 1. Resumen del progreso del total de los ejercicios en la primera etapa de entrenamiento Fuente: El presente estudio

En la figura anterior se observa que al principio de la experimentación la persona tenía que hacer más intentos para lograr el objetivo, lo que se considera comprensible debido a que estaba en etapa de aprendizaje y era poco probable que respondiera efectivamente con todas las contracciones en la primera vez. Sin embargo, a medida que la persona va practicando 
el ejercicio, vemos que en los movimientos 4 y 5 los intentos cada vez van disminuyendo. De acuerdo con los datos obtenidos, se determinó una eficacia del $40 \%$.

Durante el desarrollo de la fase 2 se evidencia un buen manejo en el control del dispositivo, pues la persona solo comete un error en el movimiento número 4, debido a una distracción momentánea. En la siguiente figura se observa que el aprendizaje respecto a la fase 1 se ha mantenido y se considera como bueno, dado que la eficacia aumentó al $80 \%$.

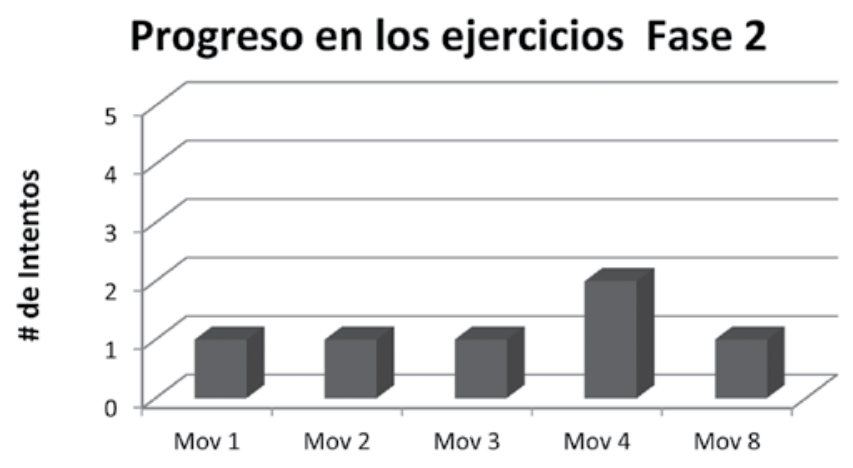

Figura 2. Resumen del progreso del total de los ejercicios en la fase 2

Fuente: El presente estudio

\section{Conclusiones}

Se logró diseñar en un ambiente virtual la representación del miembro superior derecho y controlarlo con señales mioeléctricas, para que las personas con este tipo de amputación puedan ejercitar el miembro afectado de una manera fácil y práctica. El ambiente virtual utilizado fue un entorno de realidad virtual que trae incluido Matlab, en el cual se pudo crear una interfaz interactiva y evaluar en un ambiente de aprendizaje.

Se logró representar gráficamente el miembro superior derecho de una persona en un programa de realidad virtual para llevar a cabo una simulación de cinco movimientos de la mano humana que son controlados por impulsos eléctricos provenientes del brazo de una persona.

El ambiente de aprendizaje por observación se evaluó en cada una de sus fases con cinco ejercicios de prensión, en los que básicamente se medía la eficacia del aprendizaje mediante la relación entre el porcentaje total de contracciones 
elaboradas y el porcentaje de contracciones a elaborar. Como resultado, en la fase 1 se logró una eficacia del $40 \%$ y en la fase 2 , del $80 \%$.

Con estos resultados se ve claramente que en el paso de una fase a otra existe un aumento, lo que comprueba que después de un corto entrenamiento en la interfaz, la persona al mejorar la eficacia está evidenciando la evolución de su aprendizaje. En estas condiciones se coincide con el planteamiento hecho por Sicilia y Manuel (2007), cuando argumentan que el aprendizaje en general supone un proceso de modificación de la conducta, como consecuencia de la práctica.

De acuerdo con las pruebas realizadas al circuito, se tuvo como resultado que todas las personas que se conectaron los electrodos e hicieron los diez movimientos y la persona amputada en la experimentación acusaron cansancio en el transcurso del ejercicio. Coincidieron en que este es bueno para ejercitar el músculo, pues en su mayoría sintieron dolor muscular al siguiente día de la prueba. Esto hace pensar que si el ejercicio fuera continuo y rutinario podría ser un buen método para ejercitar el miembro de la persona amputada y poder tener efectivamente un mayor control de los dispositivos externos controlados por este tipo de señales.

La representación del sistema óseo del miembro superior derecho no fue la mejor idea, pues en la experimentación, a la persona le pareció muy impactante la imagen y sugirió que fuera cambiada. Si se quiere avanzar en el desarrollo del entrenador, este debe representar el sistema tegumentario de la mano, para que tenga una apariencia más realista y la persona se sienta más cómoda con la interfaz.

El mundo virtual representado en Matlab fue de gran ayuda para retroalimentar el control que se tenía en cada contracción. Sin embargo, el tiempo de respuesta era bastante largo y esto generaba distracción en el ejercicio, para lo cual es bueno buscar un comando o una rutina en Matlab, que reduzca el tiempo de lectura del puerto, de manera que cuando el DSPic envíe los datos, inmediatamente el programa elaborado en Matlab los reconozca y la ejecución del movimiento sea más rápida, con lo que se ganaría habilidad en el aprendizaje de las rutinas motoras preestablecidas.

La memorización de los ejercicios de prensión y el número de contracciones se llevó a cabo cuando la persona entró en la fase 2. Solo en este instante se produjo la apropiación de la información pues sintió la ausencia de la hoja de referencia y se preocupó por recordar los movimientos v/s el número de contracciones. El experimentador apenas tuvo que apoyarlo en un movimiento de prensión. En el resto del proceso no tuvo sino un error.

De este resultado se desprende la idea que tiene Rosenzweig (1997) sobre el aprendizaje y la memoria, cuando dice que cada vez que la persona aprende 
algo, se necesita una memoria para evocar lo aprendido, que es lo que se refleja en el protocolo verbal de la fase de movimientos autónomos.

En el proceso de aprendizaje, la persona iba evaluando las diferentes formas de hacer una contracción válida. Al buscar una rutina estándar para todas las contracciones, la que más le funcionó fue hacer las contracciones mientras miraba el LEd, y luego de fijarse que todas estaban bien hechas, mirar la interfaz para corroborar que el movimiento sí era el elegido. Esto, en primer lugar, confirma la importancia que tiene el LEd indicador y en segundo lugar, ratifica que la persona utiliza bien el modelo teórico de aprendizaje motor correspondiente al procesamiento de la información (Páez, 2008), pues primero la persona se tomaba el tiempo para hacer un análisis perceptivo de estímulos, evidenciado cuando analizaba el ejercicio a ejecutar de tal manera que lo representaba con la mano izquierda, y luego, de acuerdo con la información recibida, daba orientación a la posible respuesta (decisional).

Lo anterior se hacía más notorio cuando decía en voz alta el número de contracciones que tenía que hacer. Por último, el mecanismo de ejecución (en este caso, los músculos) coordinaba las acciones pertinentes para llevar a cabo el movimiento.

\section{Referencias bibliográficas}

Aldana, A. (2006). Obtención y análisis de señales bioeléctricas, apoyados en el diseño e implementación de un electromiógrafo virtual. Bogotá: Universidad Pedagógica Nacional.

Alonso, A. A. Hornero, R., Espino, P., De la Rosa, S. \& Liptak L. (2002). Entrenador mioeléctrico de prótesis para amputados de brazo y mano. Revista MAPFRE de Medicina,13(1). Madrid: Universidad de Valladolid.

Antonio, O. S. \& Martínez, M. (1965). Comportamiento motor. Modelos actuales. Su aplicación al aprendizaje de habilidades en el aula de educación física, [en línea]. Madrid. Disponible en: www.edufi.ucr.ac.cr/pdf/ing/artl.pdf [2011, 29 de diciembre].

Barrientos, A. (1997). Fundamentos de robótica. Madrid: Universidad Politécnica de Madrid. McGraw-Hill.

Bernaldo, J. (1987). Lenguaje, Aprendizaje y Psicomotricidad. Buenos Aires: Médica Panamericana.

Centro de Investigaciones de la Universidad Distrital Francisco José de Caldas. (2001). Sistema electrónico de señales mioeléctricas para el control de prótesis mioeléctrica de mano. Bogotá: Universidad Distrital Francisco José de Caldas. 
Craig, J. J. (2006). Robótica. Ciudad de México: Prentice Hall.

Departamento Administrativo Nacional de Estadística, DANE. (2005). Censo general.

Driessen, P. (2003). The Experimental Portable EEG/EMG Amplifier. Victoria, BC, Canadá: University of Victoria.

Fonseca, V. Da. (2004). Psicomotricidad: Paradigmas del estudio del cuerpo y de la motricidad humana. Ciudad de México: Trillas.

García González, M. T.et al (1998). Potenciales bioeléctricos: Origen y registro. Ciudad de México: Universidad Autónoma Metropolitana Iztapalapa México. González, R. (1997). Rehabilitación Médica. Barcelona: Masson.

Guzmán, A. \& Torres, V. (2007). Pinzas y agarres privilegiables según perfil ocupacional en el diseño de una mano robótica. Manuscrito en preparación.

Harvey, D. \& Longstaff, B. (2001). The Development of a Prosthetic Arm. Adelaide: The University of Adelaide.

Hoboken N. J.: IEEE Press Editorial Board.

Maldonado, L. F. (2001). Análisis de Protocolos: Posibilidad metodológica para el estudio de procesos cognitivos. Bogotá: Universidad Pedagógica Nacional.

Merletti, R. (2004). Electromyography: Physiology, engineering and noninvasive applications.

Miralles, R. (2001) Valoración del daño corporal en el aparato locomotor. Barcelona: Masson.

Muñoz, E. (2003). Aplicaciones de las Señales Mioeléctricas para el Control de Interfaces Hombre-máquina. Popayán: Universidad del Cauca.

Orsborn, A. kirsch R.(2006). Simulation of an Above-Elbow Myoelectric Prosthetic Arm for Development of an Implanted Myoelectric Control System. Cleveland, $\mathrm{OH}$ : Western Reserve University.

Páez, J. (2008). Aprendizaje de Actividades Motoras en Procesos Prensiles con el uso de una Prótesis Mecatrónica Controlada por Señales Mioeléctricas en Personas Amputadas entre Codo y Muñeca. Bogotá: Universidad Pedagógica Nacional.

Prieto S., M. D. (1992). La modificabilidad estructural cognitiva y el programa de enriquecimiento instrumental de R. Feuerstein. Madrid: Bruño.

Rosenzweig, M. (1997). Psicología fisiológica. Madrid: McGraw-Hill. 


\section{William David Vargas, Pablo Alexander Munévar y John Jairo}

Entrenador virtual para el aprendizaje de rutinas motoras, en personas con ausencia de un miembro superior, artículo producto de la investigación

Sarmiento, L. C. (2007). Diseño de una prótesis para personas amputadas de mano y muñeca. Bogotá: Universidad Nacional de Colombia.

Suárez, R. (2007). Prensión de objetos en Robótica: Barcelona: Universidad Politécnica de Cataluña.

U. S. Department of Health and Human Services (1992). Selected Topics in Surface. National Institute for Occupational Safety and Health (1992). Electromyography for use in the Occupational Setting: Expert Perspectives.

Vivas, A. \& Aguilar, E. (2007). Modelado Geométrico y Dinámico de una Prótesis de Mano Robótica. Popayán: Universidad del Cauca.

Wallon, H. (1997). Los estadios en la psicología del niño. Buenos Aires: Ediciones nueva visión.

Wilson, F. (1998). The Hand: How Its Use Shapes the Brain, Language, and Human Culture. Barcelona: Tusquets Editores. 